\title{
Structural-Parametric Model of Electro Elastic Actuator for Nanotechnology and Biotechnology
}

\author{
Afonin SM* \\ Department of Intellectual Technical Systems, 124498, Moscow, Russia
}

*Corresponding author: Afonin SM, National Research University of Electronic Technology (MIET), Department of Intellectual Technical Systems, 124498, Moscow, Russia, E-mail: learner01@mail.ru

\begin{abstract}
:
The generalized parametric structural schematic diagram, the generalized matrix equation for the electro elastic actuator for the nanotechnology and the biotechnology are obtained. The deformations of the electro elastic actuator are described by the matrix equation. The structural-parametric model and the parametric structural schematic diagram of the electro elastic actuator or the piezoactuator are determined in contrast the electrical equivalent circuit types Cady or Mason for the calculation of the piezoelectric transmitter and receiver, the vibration piezomotor with the mechanical parameters in form the velocity and the pressure. The parametric structural schematic diagram of electro elastic actuator is obtained with the mechanical parameters the displacement and the force. The transfer functions of the electro elastic actuator are determined. The method of mathematical physics is used.
\end{abstract}

Keywords: Electro elastic actuator; Piezoactuator; Structural-parametric model; Parametric structural schematic diagram; Transfer function.

\section{Introduction}

The electro elastic actuator for the nanotechnology and the biotechnology is used in the scanning tunneling microscopes, the scanning force microscopes, the atomic force microscopes $^{[1-19]}$.

The nano- and micromanipulators with the electro elastic actuators are part of the equipment of the precision engineering, the adaptive optics and the medical surgical manipulators. The piezoactuators are used in the medical technology for the accurate instrument delivery, in the laser equipment for the controlling radiation power and the beam divergence in the optical beam scanning systems ${ }^{[1-8,14]}$.

The piezoactuators are used for the nanodisplacement in the spectrometers, the tunnel microscopes, the interferometers, where the high positioning accuracy and the parallelism of the elements displacement are required simultaneously with the small ranges of the displacements.

As the result of the joint solution of the wave equation of the electro elastic actuator with the Laplace transform, the equation of the electro elasticity and the boundary conditions on the two loaded working surfaces of the electro elastic actuator, we obtain the corresponding structural-parametric model and the parametric structural schematic diagram of the electro elastic actuator

The parametric structural schematic diagram of the electro elastic actuator on the piezoelectric, piezomagnetic, electrostriction effects, for example, the piezoactuator is determined in contrast electrical equivalent circuit types Cady or Mason for the calculation of the piezotransmitter and piezoreceiver, the vibration piezomotor with the mechanical parametres in form the velocity and the pressure ${ }^{[1-8]}$. The parametric structural schematic diagram of the actuator is obtained with the mechanical parameters the displacement and the force.

The method of mathematical physics is applied for the solution of the wave equation of the electro elastic actuator for the nanotechnology and biotechnology with using the Laplace transform for the construction the parametric structural schematic diagram of electro elastic actuator.

The parametric structural schematic diagram and the matrix transfer functions of the electro elastic actuator for the nanotechnology and biotechnology are obtained from the structural-parametric model of the electro elastic actuator with the mechanical parameters the displacement and the force. The parametric structural schematic diagrams of the voltage-controlled or current-controlled piezoactuator are determined from the generalized structural-parametric model of the actuator.

Received date: May 4, 2018

Accepted date: May 23, 2018

Publish date: May 28, 2018

Citation: Afonin, S.M. Structural-Parametric Model of Electro Elastic Actuator for Nanotechnology and Biotechnology. (2018) J Pharm Pharmaceutics 5(1): 8-12.

Copyright: (C) 2018 Afonin, S.M. This is an Open access article distributed under the terms of Creative Commons Attribution 4.0 International License. 
Electro Elastic Actuator for Nanotechnology and Biotechnology

\section{Parametric structural schematic diagram}

For the determination of the structural-parametric model and the parametric structural schematic diagram of the electroelastic actuator let us consider the generalized equation of the electroelasticity ${ }^{[8,11,18]}$ in the form

$S_{i}=d_{m i} \Psi_{m}(t)+s_{i j}^{\Psi} T_{j}(x, t)$

where $S_{i}=\partial \xi(x, t) / \partial x$ is the relative displacement along axis $i$ of the cross section of the piezoactuator or the piezoplate, $\Psi_{m}=\left\{E_{m}, D_{m}\right.$ is the control parameter $E$ for the voltage control, $D$ for the current control along axis $m, T_{j}$ is the mechanical stress along axis $j$, $d_{m i}$ is the coefficient of electro elasticity, for example, the piezo-


$\Psi=$ const, the indexes $i, j=1,2, \ldots, 6 ; m=1,2,3$.

The main size is determined us the working length for the electro elastic actuator or the piezoactuator in form the thickness, the height and the width for the longitudinal, transverse and shift piezo effect.

For the construction the parametric structural schematic diagram of electro elastic actuator in nanotechnology is used the wave equation ${ }^{[8,11,18]}$ for the wave propagation in a long line with damping but without distortions.

With using Laplace transform is obtained the linear ordinary second-order differential equation with the parameter $p$. correspondingly the original problem for the partial differential equation of hyperbolic type using the Laplace transform is reduced to the simpler problem ${ }^{[8,11,12]}$ for the linear ordinary differential equation

$$
\frac{d^{2} \Xi(x, p)}{d x^{2}}-\gamma^{2} \Xi(x, p)=0
$$

with its solution

$$
\Xi(x, p)=C e^{-x \gamma}+B e^{x \gamma}
$$

where $\Xi(x, p)$ is the Laplace transform of the displacement of section of the actuator, $\gamma=p / c^{\Psi}+\alpha$ is the propagation coefficient, $c^{\Psi}$ is the sound speed for the control parameter $\Psi=$ const, $\alpha$ is the damping coefficient, $C$ and $B$ are constants.

The generalized structural-parametric model and the generalized parametric structural schematic diagram of the electro elastic actuator the nanotechnology and the biotechnology on Figure 1 are determined, using equation of the electro elastic actuator (1) and the linear ordinary differential equation (2), the boundary conditions on loaded faces and the strains along the axes, in the following form

$$
\begin{aligned}
& \Xi_{1}(p)=\left[1 /\left(M_{1} p^{2}\right)\right] \times \\
& \times\left\{F_{1}(p)+\left(1 / \chi_{i j}^{\Psi}\right)\left[v_{m i} \Psi_{m}(p)-\left[\gamma / \operatorname{sh}\left(l_{l}\right)\right]\left[\operatorname{ch}\left(l_{1}\right) \Xi_{1}(p)-\Xi_{2}(p)\right]\right\}\right. \\
& \Xi_{2}(p)=\left[1 /\left(M_{2} p^{2}\right)\right] \times \\
& \times\left\{-F_{2}(p)+\left(1 / \chi_{i j}^{\Psi}\right)\left[v_{m i} \Psi_{m}(p)-\left[\gamma / \operatorname{sh}\left(l_{\gamma}\right)\right]\left[\operatorname{ch}\left(l_{i}\right) \Xi_{2}(p)-\Xi_{1}(p)\right]\right\}\right.
\end{aligned}
$$

where $\chi_{i j}^{\Psi}=\frac{s_{i j}^{\Psi}}{S_{0}}, v_{m i}=\left\{\begin{array}{l}d_{33}, d_{31}, d_{15} \\ g_{33}, g_{31}, g_{15}\end{array}, \Psi_{m}=\left\{\begin{array}{l}E_{3}, E_{3}, E_{1} \\ D_{3}, D_{3}, D_{1}\end{array}\right.\right.$, $\mathrm{s}_{i j}^{\Psi}=\left\{\begin{array}{l}s_{33}^{E}, s_{11}^{E}, s_{55}^{E} \\ s_{33}^{D}, s_{11}^{D}, s_{55}^{D}\end{array}, l=\left\{\delta, h, b, c^{\Psi}=\left\{c^{E}, c^{D}, \gamma^{\Psi}=\left\{\gamma^{E}, \gamma^{D}\right.\right.\right.\right.$,

$v_{m i}$ is the coefficient of the electro elasticity, for example, $d_{m i}$ is the piezomodule for the voltage-controlled piezoactuator, $g_{m i}$ is the piezomodule for the current-controlled piezoactuator, $S_{0}$ is the cross section area and $M_{1}, M_{2}$ are the displaced mass on the faces of the electro elastic actuator, $\Xi_{1}(p), \Xi_{2}(p)$ and $F_{1}(p), F_{2}(p)$ are the Laplace transform of the displacements and the forces on the faces of the electro elastic actuator, $\Psi_{m}$ is the control parameter of the electro elastic actuator.

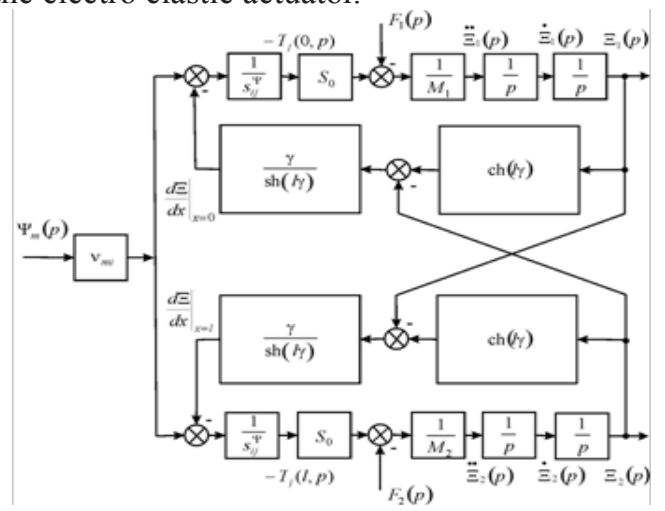

Figure 1: Generalized parametric structural schematic diagram of electro elastic actuator in nanotechnology.

The matrix state equations ${ }^{[2,11,14]}$ for the piezoelectric effect have the form

$(\mathbf{D})=(\mathbf{d})(\mathbf{T})+\left(\boldsymbol{\varepsilon}^{\mathrm{T}}\right)(\mathbf{E})$

$(\mathbf{S})=\left(\mathbf{s}^{\mathrm{E}}\right)(\mathbf{T})+(\mathbf{d})^{\mathrm{t}}(\mathbf{E})$

where the first equation describes the direct piezoeffect, the second equation presents the inverse piezoeffect, (D) is the column - matrix of the electric induction along the coordinate axes, (S) is the column-matrix of the relative deformations, (T) is the column - matrix of the mechanical stresses, (E) is the column matrix of the electric field strength along the coordinate axes, $(\mathbf{d})^{\mathrm{t}}$ is the transposed matrix of the piezoelectric modules, $\left(\mathbf{s}^{\mathrm{E}}\right)$ is the elastic compliance matrix, $\left(\varepsilon^{\mathrm{T}}\right)$ is the matrix of dielectric constants.

The deformation of the piezoactuator corresponds to its stressed state. If the mechanical stress $T$ are created in the piezoactuator, the deformations $S$ are formed in the piezoactuator ${ }^{[8,11]}$.

There are the six stress components $T_{1}, T_{2}, T_{3}, T_{4}, T_{5}$, $T_{6}$. The components $T_{1}-T_{3}$ are defined to extension-compression stresses, the components $T_{4}-T_{6}$ are related to shear stresses.

Let us consider the transverse piezoelectric effect in the piezoactuator. The equation of the inverse transverse piezoeffect ${ }^{[8,11]}$ in the piezoactuator can be written in the following form $S_{1}=d_{31} E_{3}(t)+s_{11}^{E} T_{1}(x, t)$

where $S_{1}=\frac{\partial \xi(x, t)}{\partial x}$ is the relative displacement of the cross section of the plezoactuator along axis $1, d_{31}$ is the piezoelectric module for the transverse piezoeffect, $s_{11}^{E}$ is the elastic compliance for $E=$ const along axis $1, T_{1}$ is the stress along axis 1 .

The solution of the linear ordinary second-order differential equation with the parameter $p(2)$ can be written as (3) and subject to the conditions. 
Citation: Afonin, S.M. Structural-Parametric Model of Electro Elastic Actuator for Nanotechnology and Biotechnology. (2018) J Pharm Pharmaceutics 5(1): 8-12.

$$
\begin{aligned}
& \Xi(0, p)=\Xi_{1}(p) \text { For } x=0, \\
& \Xi(h, p)=\Xi_{2}(p) \text { For } x=h
\end{aligned}
$$

Therefore the constants $C$ and $B$ for the solution we obtain in the following form

$$
C=\left(\Xi_{1} e^{h_{\gamma}}-\Xi_{2}\right) /\left[2 \operatorname{sh}\left(h_{\gamma}\right)\right], B=\left(\Xi_{2}-\Xi_{1} e^{-h_{l}}\right) /\left[2 \operatorname{sh}\left(h_{\gamma}\right)\right]
$$

Then, the solution of the linear ordinary second-order differential equation (2) in form (3) can be written as

$$
\exists(x, p)=\left\{\Xi_{1}(p) \operatorname{sh}[(h-x) \gamma]+\Xi_{2}(p) \operatorname{sh}(x \gamma)\right\} / \operatorname{sh}(h \gamma)
$$

The equations of forces acting on the faces of the piezoactuator has the form

$T_{1}(0, p) S_{0}=F_{1}(p)+M_{1} p^{2} \Xi_{1}(p)$ For $x=0$

$T_{1}(h, p) S_{0}=-F_{2}(p)-M_{2} p^{2} \Xi_{2}(p)$ For $x=h$

where $T_{1}(0, p)$ and $T_{1}(h, p)$ are determined from the equation of the inverse transverse piezoeffect. Therefore we obtain the system of the equations for the mechanical stresses at the faces of the actuator for the transverse piezoeffect in the form

$$
\begin{aligned}
& T_{1^{\prime}}(0, p)=\left.\frac{1}{s_{11}^{E}} \frac{d \Xi(x, p)}{d x}\right|_{x=0}-\frac{d_{31}}{s_{11}^{E}} E_{3}(p) \\
& T_{1}(h, p)=\left.\frac{1}{s_{11}^{E}} \frac{d \Xi(x, p)}{d x}\right|_{x=h}-\frac{d_{31}}{s_{11}^{E}} E_{3}(p)
\end{aligned}
$$

The set of equations (12) for mechanical stresses in piezoactuator yields the following set of equations describing the structural parametric model and parametric structural schematic diagram of the voltage-controlled piezoactuator for the transverse piezoelectric effect on Figure 2.

$$
\begin{aligned}
& \Xi_{1}(p)=\left[1 /\left(M_{1} p^{2}\right)\right] \times \\
& \times\left\{-F_{1}(p)+\left(1 / \chi_{11}^{E}\right)\left[d_{31} E_{3}(p)-[\gamma / \operatorname{sh}(h \gamma)]\left[\operatorname{ch}(h \gamma) \Xi_{1}(p)-\Xi_{1}(p)\right]\right\}\right. \\
& \Xi_{2}(p)=\left[1 /\left(M_{2} p^{2}\right)\right] \times \\
& \times\left\{-F_{2}(p)+\left(1 / \chi_{11}^{E}\right)\left[d_{31} E_{3}(p)-[\gamma / \operatorname{sh}(h \gamma)]\left[\operatorname{ch}(h \gamma) \Xi_{2}(p)-\Xi_{1}(p)\right]\right\}\right.
\end{aligned}
$$

where $\chi_{11}^{E}=\frac{s_{11}^{E}}{s_{\mathrm{o}}}, l=h$.



Figure 2: Parametric structural schematic diagram of voltage-controlled piezoactuator for transverse piezoelectric effect.

The parametric structural schematic diagrams of the voltage-controlled or current-controlled piezoactuator for the transverse, longitudinal, shift piezoelectric effects are deter- mined from the generalized structural-parametric model of the electro elastic actuator.

\section{Transfer functions}

From generalized structural-parametric model of the electro elastic actuator, taking into account the generalized equation of the electro elasticity, wave equation and the equation of the forces on its faces, we obtain the transfer functions of the electro elastic actuator. Correspondingly the Laplace transforms of displacements for two faces of the actuator are dependent from the Laplace transforms of the general parameter of control and forces on two faces

$\Xi_{1}(p)=W_{11}(p) \Psi_{m}(p)+W_{12}(p) F_{1}(p)+W_{13}(p) F_{2}(p)$

$\Xi_{2}(p)=W_{21}(p) \Psi_{m}(p)+W_{22}(p) F_{1}(p)+W_{23}(p) F_{2}(p)$

Matrix equation of the Laplace transforms of the displacements with the matrix transfer functions of the electro elastic actuator is obtained ${ }^{[8,14,18]}$ in the form

$\left(\begin{array}{l}\Xi_{1}(p) \\ \Xi_{2}(p)\end{array}\right)=\left(\begin{array}{lll}W_{11}(p) & W_{12}(p) & W_{13}(p) \\ W_{21}(p) & W_{22}(p) & W_{23}(p)\end{array}\right)\left(\begin{array}{c}\Psi_{m}(p) \\ F_{1}(p) \\ F_{2}(p)\end{array}\right)$

Let us consider the displacements the faces of the voltage-controlled the piezo actuator for the transverse piezoeffect $\Psi_{m}=E_{3}$ with the output parameter displacement. The transfer functions of the voltage-controlled the piezoactuator for the transverse piezoeffect can be written in the form

$$
\begin{aligned}
& W_{11}(p)=\Xi_{1}(p) / E_{3}(p)=d_{31}\left[M_{2} \chi_{11}^{E} p^{2}+\gamma \operatorname{th}(h / / 2)\right] / A_{11} \\
& \chi_{11}^{E}=s_{11}^{E} / s_{0}
\end{aligned}
$$$$
A_{11}=M_{1} M_{2}\left(\chi_{11}^{E}\right)^{2} p^{4}+\left\{\left(M_{1}+M_{2}\right) \chi_{11}^{E} /\left[c^{E} \operatorname{th}(h \gamma)\right] p^{3}+\right.
$$$$
+\left[\left(M_{1}+M_{2}\right) \chi_{11}^{E} \alpha / \operatorname{th}(h \gamma)+1 /\left(c^{E}\right)^{2}\right] p^{2}+2 \alpha p / c^{E}+\alpha^{2}
$$$$
W_{21}(p)=\Xi_{2}(p) / E_{3}(p)=d_{31}\left[M_{1} \chi_{11}^{E} p^{2}+\gamma \operatorname{th}(h \gamma / 2)\right] / A_{11}
$$$$
W_{12}(p)=\Xi_{1}(p) / F_{1}(p)=-\chi_{11}^{E}\left[M_{2} \chi_{11}^{E} p^{2}+\gamma / \operatorname{th}(h \gamma)\right] / A_{11}
$$$$
W_{13}(p)=\Xi_{1}(p) / F_{2}(p)=
$$$$
=W_{22}(p)=\Xi_{2}(p) / F_{1}(p)=\left[\chi_{11}^{E} / \operatorname{sh}\left(h_{Y}\right)\right] / A_{11}
$$

$$
W_{23}(p)=\Xi_{2}(p) / F_{2}(p)=-\chi_{11}^{E}\left[M_{1} \chi_{11}^{E} p^{2}+\gamma / \operatorname{th}(h \gamma)\right] / A_{11}
$$

The static displacement of the faces the electro elastic actuator $\xi_{1}(\infty)$ and $\xi_{2}(\infty)$ can be written in the form

$$
\begin{aligned}
& \xi_{1}(\infty)=\lim _{t \rightarrow \infty} \xi_{1}(t)=\frac{v_{m i} l \Psi_{m 0}\left(M_{2}+m / 2\right)}{M_{1}+M_{2}+m} \\
& \xi_{2}(\infty)=\lim _{t \rightarrow \infty} \xi_{2}(t)=\frac{v_{m i} l \Psi_{m 0}\left(M_{1}+m / 2\right)}{M_{1}+M_{2}+m} \\
& \xi_{1}(\infty)+\xi_{2}(\infty)=\lim _{t \rightarrow \infty}\left(\xi_{1}(t)+\xi_{2}(t)\right)=v_{m i} l \Psi_{m 0}
\end{aligned}
$$

where $m$ is the mass actuator, $M_{1}, M_{2}$ are the load masses.

Let us consider example at $m<<M_{1}$ and $m<<M_{2}$ for the voltage-controlled the piezoactuator from PZT under the longitudinal piezoeffect $d_{33}=4 \cdot 10^{-10} \mathrm{~m} / \mathrm{V}, U=150 \mathrm{~V}, M_{1}=1 \mathrm{~kg}$ and $M_{2}=4 \mathrm{~kg}$ we obtain the static displacements of the faces of the piezo actuator $\xi_{1}(\infty)=48 \mathrm{~nm}, \xi_{2}(\infty)=12 \mathrm{~nm}, \xi_{1}(\infty)+\xi_{2}(\infty)=60 \mathrm{~nm}$. 
The static displacements of the faces for the voltage-controlled the piezoactuator for the transverse piezoeffect are obtained from (15) at $m<<M_{1}$ and $m<<M_{2}$ in the form

$$
\begin{aligned}
& \xi_{1}(\infty)=\lim _{\substack{p \rightarrow 0 \\
\alpha \rightarrow 0}} \frac{p W_{11}(p) U_{0}}{\delta p}=\frac{d_{31} h U_{0} M_{2}}{\delta\left(M_{1}+M_{2}\right)} \\
& \xi_{2}(\infty)=\lim _{\substack{p \rightarrow 0 \\
\alpha \rightarrow 0}} \frac{p W_{21}(p) U_{0}}{\delta p}=\frac{d_{31} h U_{0} M_{1}}{\delta\left(M_{1}+M_{2}\right)}
\end{aligned}
$$

For the voltage-controlled the piezoactuator from PZT under the transverse piezoeffect at $m<<M_{1}$ and $m<<M_{2}, d_{31}$ $=2 \cdot 10^{-10} \mathrm{~m} / \mathrm{V}, h / \delta=20, U=100 \mathrm{~V}, M_{1}=2 \mathrm{~kg}$ and $M_{2}=8 \mathrm{~kg}$ the static displacements of the faces the piezoactuator are determined $\xi_{1}(\infty)=320 \mathrm{~nm}, \xi_{2}(\infty)=80 \mathrm{~nm}, \xi_{1}(\infty)+\xi_{2}(\infty)=400 \mathrm{~nm}$.

The transfer function of the voltage-controlled transverse piezoactuator is obtained from (15) for elastic-inertial load at $M_{1} \rightarrow \infty, m<<M_{2}$ and the approximation the hyperbolic cotangent by two terms of the power series in the form

$$
\begin{aligned}
& W(p)=\frac{\Xi_{2}(p)}{U(p)}=\frac{d_{31} h / \delta}{\left(1+C_{e} / C_{11}^{E}\right)\left(T_{t}^{2} p^{2}+2 T_{t} \xi_{t} p+1\right)} \\
& T_{t}=\sqrt{M_{2} /\left(C_{e}+C_{11}^{E}\right)}, \xi_{t}=\alpha h^{2} C_{11}^{E} /\left(3 c^{E} \sqrt{M_{2}\left(C_{e}+C_{11}^{E}\right)}\right)
\end{aligned}
$$

where the Laplace is transform of the voltage, $T_{t}$ is the time constant and $\xi_{t}$ is the damping coefficient of the piezoactuator. Therefore the expression for the transient response of the voltage-controlled transverse piezoactuator is determined in the following form

$$
\begin{aligned}
& \xi(t)=\xi_{m}\left[1-\frac{e^{-\frac{\xi_{t} t}{T_{t}}}}{\sqrt{1-\xi_{t}^{2}}} \sin \left(\omega_{t} t+\varphi_{t}\right)\right] \\
& \xi_{m}=\frac{d_{31}(h / \delta) U_{m}}{1+C_{t} / C_{11}^{E}}, \omega_{t}=\frac{\sqrt{1-\xi_{t}^{2}}}{T_{t}}, \varphi_{t}=\operatorname{arctg}\left(\frac{\sqrt{1-\xi_{t}^{2}}}{\xi_{t}}\right)
\end{aligned}
$$

where $\xi_{m}$ is the steady-state value of displacement for the voltage-controlled piezoactuator, $U_{m}$ is the amplitude of the voltage in the steady-state. For $M_{1} \rightarrow \infty, m<<M_{2} \cdot U_{\mathrm{m}}=50 \mathrm{~V}, d_{31}=2 \cdot 10^{-10}$ $\mathrm{m} / \mathrm{V}, h / \delta=20, M_{2}=4 \mathrm{~kg}, C_{11}^{E}=2.4 \cdot 10^{7} \mathrm{H} / \mathrm{m}, C_{e}=0.1 \cdot 10^{7} \mathrm{H} / \mathrm{m}$ we obtain values the steady-state value of displacement and the time constant of the actuator $\xi_{m}=192 \mathrm{~nm}, T_{t}=0.4 \cdot 10^{-3} \mathrm{c}$.

The matrix transfer functions of the actuator are determined for control systems with the electro elastic actuator for the nanotechnology and the biotechnology.

In this work the generalized parametric structural schematic diagram and generalized structural-parametric model of the electro elastic actuator are obtained. From generalized structural-parametric model of the electro elastic actuator after algebraic transformations the transfer functions of the electro elastic actuator are determined. The parametric structural schematic diagrams, the structural-parametric models of the piezoactuator for the transverse, longitudinal, shift piezoelectric effects are determined from the generalized structural-parametric model of the electro elastic actuator for the nanotechnology and the biotechnology.

\section{Conclusions}

For the nanotechnology and the biotechnology the generalized parametric structural schematic diagram and the generalized structural-parametric model of the electro elastic actuator are constructed with the mechanical parameters the displacement and the force.

The parametric structural schematic diagrams of the piezoactuator for the transverse, longitudinal, shift piezoelectric effects are determined.

The matrix transfer functions of the electro elastic actuator are determined in the control systems for the nanotechnology and the biotechnology. 
Citation: Afonin, S.M. Structural-Parametric Model of Electro Elastic Actuator for Nanotechnology and Biotechnology. (2018) J Pharm Pharmaceutics 5(1): 8-12.

\section{References}

1. Schultz, J., Ueda, J., Asada, H. Cellular actuators. (2017) Oxford: Butterworth-Heinemann 382.

Pubmed | Crossref | Others

2. Afonin, S.M. Absolute stability conditions for a system controlling the deformation of an elecromagnetoelastic transduser. (2006) Doklady mathematics 74(3): 943-948.

Pubmed |Crossref | Others

3. Zhou, S., Yao, Z. Design and optimization of a modal - independent linear ultrasonic motor. (2014) IEEE transaction on ultrasonic's, ferroelectrics and freq control 61(3): 535-546.

Pubmed $\mid$ Crossref $\mid$ Others

4. Przybylski, J., Static and dynamic analysis of a flex tensional transducer with an axial piezoelectric actuation. (2015) Engineering structures 84: 140-151.

Pubmed | Crossref | Others

5. Ueda, J., Secord, T., Asada, H. H. Large effective-strain piezoelectric actuators using nested cellular architecture with exponential strain amplification mechanisms. (2009) IEEE/ASME transactions on mechatronics15 (5): 770-782. Pubmed |Crossref | Others

6. Karpelson, M., Wei, G-Y., Wood, RJ. Driving high voltage piezoelectric actuators in micro robotic applications. (2012) Sensors and actuators A Physical 176: 78-89.

Pubmed $\mid$ Crossref $\mid$ Others

7. Afonin, S.M. Block diagrams of a multilayer piezoelectric motor for nano- and microdisplacements based on the transverse piezoeffect.(2015) Journal of computer and systems sciences international 54(3): 424-439.

Pubmed |Crossref | Others

8. Afonin, S.M. Structural parametric model of a piezoelectric nanodisplacement transduser. (2008) Doklady physics 53(3): 137.

Pubmed $\mid$ Crossref $\mid$ Others

9. Afonin, S.M. Solution of the wave equation for the control of an elecromagnetoelastic transduser. (2006) Doklady mathematics 73(2): 307-313.

Pubmed |Crossref| Others
10. Cady, W.G. Piezoelectricity: An introduction to the theory and applications of electromechancial phenomena in crystals. (1946) New York London Hill Book Company: 806.

Pubmed |Crossref | Others

11. Mason, W. P. Physical acoustics: Principles and methods. (1964) Vol.1. Part A. Methods: 532.

Pubmed | Crossref | Others

12. Zwillinger, D. Handbook of differential equations. (1989) Boston: Academic Press 673.

Pubmed |Crossref | Others

13. Afonin, S.M. Structural-parametric model and transfer functions of electroelastic actuator for nano- and microdisplacement. (2015) Piezoelectrics and nano materials New York: 225-242.

Pubmed | Crossref | Others

14. Afonin SM. A structural-parametric model of electroelastic actuator for nano- and microdisplacement of mechatronic system. (2017) Eds. Bartul Z, Trenor J. New York: 259-284. Pubmed |Crossref $\mid$ Others

15. Afonin, S.M. Nano- and micro-scale piezomotors. Russian engineering research. (2012) Russian Engineering Research 32(7-8): 519-522.

Pubmed | Crossref $\mid$ Others

16. Afonin, S.M. Elastic compliances and mechanical and adjusting characteristics of composite piezoelectric transducers. Mechanics of solids. (2007) Mechanics of Solids 42(1): 43-49.

Pubmed | Crossref $\mid$ Others

17. Afonin, S.M. Stability of strain control systems of nanoand microdisplacement piezotransducers. (2014) Mechanics of solids 49(2): 196-207.

Pubmed $\mid$ Crossref $\mid$ Others

18. Afonin, S.M. Structural-parametric model electromagnetoelastic actuator nanodisplacement for mechatronics. (2017) International journal of physics 5(1): 9-15.

Pubmed | Crossref | Others

19. Bhushan, B. Springer Handbook of Nanotechnology. (2004) Ed. $3^{\text {rd }}$ revised Springer: 1222.

Pubmed $\mid$ Crossref $\mid$ Others

Submit your manuscript to Ommega Publishers and we will help you at every step:

- We accept pre-submission inquiries

- Our selector tool helps you to find the most relevant journal

- We provide round the clock customer support

- Convenient online submission

- Thorough peer review

- Inclusion in all major indexing services

- Maximum visibility for your research

Submit your manuscript at

OMmega Publishers

https://www.ommegaonline.org/submit-manuscript 